\title{
A CD46 transgenic mouse model for studying the histopathology of arthritis caused by subcutaneous infection with Streptococcus dysgalactiae subspecies equisimilis \\ Correspondence \\ Hidenori Matsui \\ hmatsui@lisci.kitasato-u.ac.jp \\ Received 6 May 2011 \\ Accepted 12 August 2011

\author{
Haruno Yoshida, ${ }^{1}$ Hidenori Matsui, ${ }^{1}$ Somay Yamagata Murayama, ${ }^{1}$ \\ Yasunari Takada, ${ }^{2}$ Koichi Matsuo, ${ }^{2}$ Tetsufumi Takahashi, ${ }^{3}$ \\ Masahiko Nakamura, ${ }^{3}$ Kimiko Ubukata $^{1}$ and Takashi Takahashi ${ }^{1}$ \\ ${ }^{1}$ Kitasato Institute for Life Sciences and Graduate School of Infection Control Sciences, Kitasato \\ University, 5-9-1 Shirokane, Minato-ku, Tokyo 108-8641, Japan \\ ${ }^{2}$ Laboratory of Cell and Tissue Biology, School of Medicine, Keio University, 35 Shinanomachi, \\ Shinjuku-ku, Tokyo 160-8582, Japan \\ ${ }^{3}$ Center for Clinical Pharmacy and Clinical Sciences, School of Pharmaceutical Sciences, Kitasato \\ University, 5-9-1 Shirokane, Minatoku, Tokyo 108-8641, Japan
}

\begin{abstract}
Ankle arthritis was induced by a single subcutaneous (s.c.) infection of $1 \times 10^{7}$ c.f.u. of the Streptococcus dysgalactiae subspecies equisimilis strain RE378, which was isolated from a patient suffering from multiple organ failure due to septicaemia, into both hind footpads of human CD46-expressing transgenic (Tg) mice. In contrast, in non-Tg mice, the incipient foot lesions (swelling and redness) resolved before arthritis developed. The number of viable bacteria in tissue samples and the arthritis frequency on days 3 and 28 after infection were higher in CD46 Tg mice than in non-Tg mice. The histopathological findings in the hind ankle sections of CD46 Tg mice showed the stimulation of osteoclast formation associated with inflammation of the synovial membrane and the development of aggressive granulation tissue (pannus). In addition, increased expression levels of interleukin (IL)-6, receptor activator of NF- $\kappa \mathrm{B}$ ligand, IL-1 $\beta$ and tumour necrosis factor alpha were detected in the foot bones of CD46 Tg mice but not in those of non-Tg mice. These observations suggest that the s.c. infection with $S$. dysgalactiae subsp. equisimilis induced arthritis in the ankle joints of CD46 Tg mice as a consequence of the prolonged inflammation associated with focal bone loss.
\end{abstract}

\section{INTRODUCTION}

Streptococcus dysgalactiae subspecies equisimilis has Lancefield group $\mathrm{C}$ or $\mathrm{G}$ antigens, exhibits strong $\beta$-haemolysis, and exerts streptokinase activity upon human plasminogen and proteolytic activity. S. dysgalactiae subsp. equisimilis is detected primarily in sore throats and skin sores of patients. Increasing numbers of patients suffering from severe invasive infections with $S$. dysgalactiae subsp. equisimilis have recently been reported worldwide (Bramhachari et al., 2010; Rantala et al., 2010; Sylvetsky et al., 2002; Takahashi et al., 2010a, b, 2011). S. dysgalactiae subsp. equisimilis is

Abbreviations: ASS, arthritis severity score; GAPDH, glyceraldehyde-3phosphate dehydrogenase; GAS, group A streptococcus; GBS, group B streptococcus; H\&E, haematoxylin and eosin; IL, interleukin; NF, necrotizing fasciitis; OPG, osteoprotegerin; PLN, popliteal lymph node; RANK, receptor activator of NF- $\kappa B$; RANKL, RANK ligand; s.c., subcutaneous; Tg, transgenic; TNF- $\alpha$, tumour necrosis factor alpha; TRAP, tartrate-resistant acid phosphatase. now thought to cause a range of infections, from relatively mild to severe life-threatening invasive diseases, such as acute pharyngitis, pyoderma, cellulitis, wound infections, abscesses, erysipelas, necrotizing fasciitis (NF), septic arthritis, vertebral osteomyelitis, septicaemia, multiple organ failure and streptococcal toxic shock syndrome (Brandt \& Spellerberg, 2009; Broyles et al., 2009; Sing et al., 2001). Many of these indications can show substantial overlap with those of patients infected with group A streptococcus (GAS). To compare the clinical features of invasive $S$. dysgalactiae subsp. equisimilis infections with those of infections caused by GAS and group B streptococcus (GBS), active surveillance was conducted for 1 year from 2006 to 2007 at 142 medical institutions throughout Japan. The total number of invasive infections was 479, including 231 (48\%), 97 (20\%) and 151 (32\%) cases of S. dysgalactiae subsp. equisimilis, GAS and GBS infection, respectively. Moreover, 23 (10\%), 3 (3.1\%) and $4(2.6 \%)$ patients infected with $S$. dysgalactiae subsp. equisimilis, GAS and GBS, respectively, were suffering from 
septic arthritis. Along these lines, it is worth noting that patients infected with $S$. dysgalactiae subsp. equisimilis present with septic arthritis more often than those infected with GAS or GBS (Takahashi et al., 2010b).

For the study of the pathogenesis and host defence mechanisms of $S$. dysgalactiae subsp. equisimilis infection, it is necessary to develop animal models that reflect the natural infection process in the human host. Transgenic (Tg) mice expressing human genes have been used as recipients in humanized mouse models for GAS infection. Mice carrying the human plasminogen (Maurer et al., 2006; Sun et al., 2004) or CD46 (also known as membrane cofactor protein, MCP) (Lövkvist et al., 2008; Matsui et al., 2009) have been shown to be more susceptible to GAS infection than non-Tg mice. Human CD46 has been reported to serve as a receptor for the bacterial cell surface $\mathrm{M}$ protein (the $\mathrm{emm}$ gene product) of GAS (Okada et al., 1994, 1995; Perez-Casal et al., 1995). GAS and S. dysgalactiae subsp. equisimilis have been shown to share many of the same virulence-related genes, including $\mathrm{emm}$ (Brandt \& Spellerberg, 2009; Davies et al., 2007; Shimomura et al., 2011). Accordingly, in the present study we aimed to develop a CD46 Tg mouse model of $S$. dysgalactiae subsp. equisimilis infection that mimics human disease. Here we show that arthritis occurs much more frequently in CD46 Tg mice than in non-Tg mice following subcutaneous (s.c.) infection with $S$. dysgalactiae subsp. equisimilis.

\section{METHODS}

Mice. Human CD46-expressing Tg mice were donated by Dr J. P. Atkinson of Washington University (St Louis, MO, USA). C57BL/6J mice, employed as the corresponding non-Tg mice, were obtained from Charles River Japan, where they were established as described previously (Matsui et al., 2009). All mice were bred at the animal facility at Kitasato University, and all mouse experiments were performed in accordance with institutional guidelines under an approved protocol.

Bacteria. S. dysgalactiae subsp. equisimilis strain RE378 (emm type stg6792.3) was isolated from a 62-year-old male patient who was suffering from multiple organ failure due to septicaemia and who died the day after he was admitted to the hospital (Koshigaya Municipal Hospital, Saitama, Japan). S. dysgalactiae subsp. equisimilis strains were routinely grown on Todd-Hewitt broth containing $0.2 \%$ $(\mathrm{w} / \mathrm{v})$ yeast extract (Difco and $\mathrm{BBL}$ ) in $5 \% \mathrm{CO}_{2}$ at $37{ }^{\circ} \mathrm{C}$ without shaking.

Infection. The hind footpads of 7- to 10-week-old male mice were subcutaneously infected with $1 \times 10^{7}$ c.f.u. per footpad of RE378 in a stationary growth phase. After s.c. infection, the survival rates were observed every day for 28 days, and the viable counts of bacteria in the liver, spleen, kidneys and popliteal lymph nodes (PLNs) were determined by plating onto sheep-blood agar (Matsui et al., 2009, 2011).

Macroscopic and microscopic observations. Macroscopic images were obtained with a digital camera (D80; Nikon). For histological examination, a portion of each foot bone was fixed with $4 \%(\mathrm{w} / \mathrm{v})$ paraformaldehyde in $0.1 \mathrm{M}$ sodium phosphate buffer $(\mathrm{pH} 7.2)$, decalcified in $0.5 \mathrm{M}$ EDTA $(\mathrm{pH} 7.0-7.5)$ for $3-5$ days, and then embedded in paraffin. Tissue sections approximately $5 \mu \mathrm{m}$ thick were prepared and mounted on glass slides. The tissues were stained with haematoxylin and eosin (H\&E) or tartrate-resistant acid phosphatase (TRAP) using an acid phosphatase leukocyte (TRAP) kit (no. 386A; Sigma-Aldrich), and scanned with a microscope (Axiovert 135; Carl Zeiss).

Evaluation of the severity of arthritis. The degrees of swelling and erythema of each infected mouse foot were scored (arthritis severity score; ASS) as follows: no swelling and no erythema (ASS $=0$ ), mild erythema (ASS $=1)$, mild swelling and moderate erythema (ASS $=2$ ), and severe swelling and severe erythema (ASS=3) (Sakurai et al., 2003).

Quantitative real-time RT-PCR analysis. After the skin, soft tissue and muscle were quickly removed from the foot, the cuneiform and metatarsal bones were partly smashed with a pestle. Total RNA was isolated from the smashed bones using Sepazol-RNA I Super G reagent (Nacalai Tesque) and purified using a FastPure RNA kit (Takara) according to the manufacturer's instructions. The purified RNA was quantified by spectrophotometry and analysed immediately by quantitative real-time RT-PCR. The PCR was performed in two steps as outlined below. First, an iScript cDNA synthesis kit (Bio-Rad Laboratories) was used to carry out the reverse transcriptase reaction with $1 \mu \mathrm{g}$ purified RNA according to the manufacturer's instructions. Second, an iQ SYBR Green Supermix (Bio-Rad Laboratories) kit was used to carry out the real-time quantitative PCR assay in triplicate, along with a primer pair for osteoprotegerin (OPG) (5'-CCTGGAGATCGAATTCTGCTTGA-3' ${ }^{\prime}$, 5' -TTTGCAAACTGTGTTTCGCTCTG-3'), interleukin (IL)-6 (5'-CCACTTCACAAGTCGGAGGCTTA-3', $5^{\prime}$-GCAAGTGCATCATCGTTGTTCATAC- $3^{\prime}$ ), receptor activator of NF- $\kappa$ B (RANK) ligand (RANKL) (5'-GCAGCATCGCTCTGTTCCTGTA-3', 5'-CCTGCAGGAGTCAGGTAGTGTGTC-3'), IL-17A (5'-GAAGGCCCTCAGACTACCTCAAC-3'， 5'-TCATGTGGTGGTCCAGCTTTC-3'), RANK (5' -TTCGTCCACAGACAAATGCAAAC-3', 5' -GCTGCAGACCACATCTGATTCC- $\left.3^{\prime}\right)$, tumour necrosis factor alpha (TNF- $\left.\alpha\right)\left(5^{\prime}\right.$ GTTCTATGGCCCAGACCCTCAC-3', $5^{\prime}$-GGCACCACTAGTTGGTTGTCTCTTTG-3'), IL-1 $\beta$ (5'-TCCAGGATGAGGACATGAGCAC-3', 5'-GAACGTCACACACCAGCAGGTTA-3') or glyceraldehyde-3-phosphate dehydrogenase (GAPDH) (5'-TGTGTCCGTCGTGGATCTGA-3', 5'-TTGCTGTTGAAGTCGCAGGAG-3') using the CFX96 real-time PCR detection system (Bio-Rad Laboratories) according to the manufacturer's instructions.

Statistics. Significant differences between the mean plus or minus SD values of different groups were examined using the two-tailed unpaired Student's $t$-test. A $P$ value of $<0.05$ was regarded as statistically significant.

\section{RESULTS}

\section{S.c. infection with RE378 induced arthritis in the feet of CD46 Tg mice}

The footpad skin of CD46 Tg and non-Tg mice exhibited swelling and redness on day 3 after s.c. infection with $1 \times 10^{7}$ c.f.u. RE378 (data not shown). As shown in Fig. 1, on day 28 after infection, CD46 Tg mice developed the macroscopic appearance of ankle arthritis, while non- $\mathrm{Tg}$ mice fully recovered from the foot injuries. Indeed, the infected CD46 $\mathrm{Tg}$ mice reached ASS $=2.9 \pm 0.3 \quad(n=12)$, whereas the infected non-Tg mice had ASS $=0.1 \pm 0.3 \quad(n=12)$. Then, we challenged CD46 Tg and non-Tg mice with s.c. infection of $1 \times 10^{7}$ c.f.u. of five other strains of $S$. dysgalactiae subsp. 
(a)
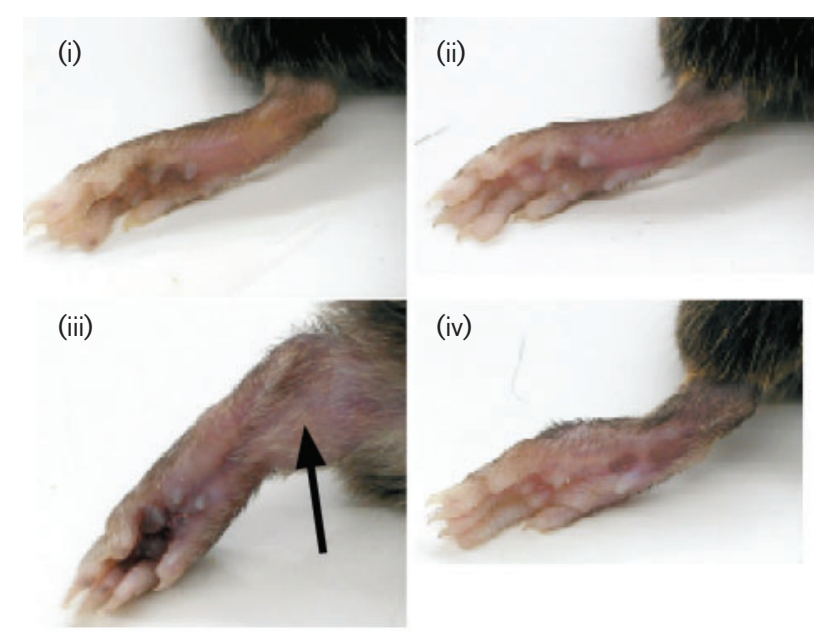

(iv)

(b)

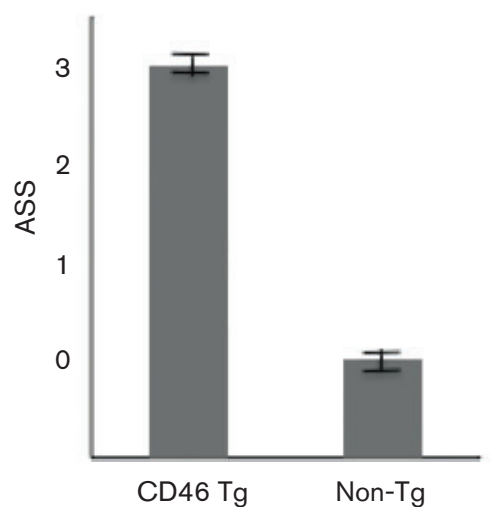

Fig. 1. Representative appearance of the feet of CD46 Tg and non-Tg mice. (a) The macroscopic appearance of the feet of a CD46 Tg (i, iii) and non-Tg (ii, iv) mouse on days 0 (i, ii) and 28 (iii, iv) after s.c. infection with $1 \times 10^{7}$ c.f.u. RE378 per footpad is shown. The arrow indicates arthritis. (b) ASSs on day 28 after infection. The data represent the mean values of the number of bacteria per tissue sample $\pm \mathrm{SD}(n=12)$.

equisimilis (emm types stG480.0, stC36.0, stG485.0, stL2764.0 and stL2764.0) isolated from human and swine into the footpads. The CD46 Tg mice infected with the virulent strains isolated from a patient and swine with invasive diseases developed severe arthritis 28 days after infection. However, the non-Tg mice infected with the virulent strains, or even the CD46 Tg mice infected with avirulent strains isolated from patients with non-invasive diseases, did not develop arthritis 28 days after infection (data not shown). These findings suggest that the development of arthritis in CD46 Tg mice induced by s.c. infection with the virulent strains of $S$. dysgalactiae subsp. equisimilis requires the presence and expression of virulence-associated genes.

\section{s.c. infection with RE378 was associated with bacterial load in PLNs}

We monitored the number of viable bacteria in the liver, spleen, kidneys and PLNs of CD46 Tg and non-Tg mice on days 3, 7 and 15 after s.c. infection with RE378. As shown in Fig. 2, less than 10, 100 or 1000 c.f.u. of bacteria per tissue sample were detected in the spleen and kidneys, liver or PLNs, respectively, of CD46 Tg mice on day 3 after s.c. infection. In contrast, in non-Tg mice, viable bacteria were detected only in the PLNs and not in any of the other tissues (liver, spleen and kidneys) at this time point. In the PLNs, there was no clear difference in the numbers of viable bacteria between CD46 Tg and non-Tg mice. In addition, no bacterial load was detected in any of the tissue samples of the CD46 Tg or non-Tg mice examined on days 7 and 15 after s.c. infection. Accordingly, all CD46 Tg and non-Tg mice survived for 28 days after s.c. infection. These results suggest that the development of arthritis in the feet of CD46 Tg mice is independent of the prolonged colonization of bacteria in the diseased part. In general, infectious arthritis can be subclassified into two types: pyogenic (septic) and non-pyogenic (Greenspan \& Tehranzadeh, 2001). Mouse models of pyogenic arthritis caused by streptococcus infection have been reported (Abdollahi-Roodsaz et al., 2008; Jonsson et al., 2005; Joosten et al., 2008; Lövkvist et al., 2008; Sakurai et al., 2003), but this is believed to be the first report of an animal model of non-pyogenic arthritis caused by s.c. infection with S. dysgalactiae subsp. equisimilis.

\section{S.c. infection with RE378 induced inflammatory arthritis in the ankles of CD46 Tg mice}

We performed histochemical analyses of H\&E and TRAP stained ankle joint sections of CD46 Tg and non-Tg mice after s.c. infection with RE378. As shown in Fig. 3(a, b, c, d) (H\&E staining), severe inflammatory cell infiltration and aggressive granulation tissue (pannus) formation were observed inside the articular cavity of the ankle joints of CD46 Tg mice on day 28 after s.c. infection with RE378 (Fig. $3 c)$. It is possible that inflammation of the synovial membrane resulted in the development of pannus. Moreover, the pannus invaded the cartilage, indicating that there was an erosion of bone cartilage (Fig. 3c). However, in the case of non-Tg mice, neither inflammatory cell infiltration nor pannus formation was induced in the feet on day 28 after s.c. infection with RE378 (Fig. 3d). In this regard, as shown in Fig. 4(a, b, c, d, e) (the TRAP stained tissue), TRAP-positive mononuclear and multinucleated cells (osteoclasts) were observed at the bone-cartilage interface in the ankle joints of CD46 Tg (Fig. 4c, e) but not non-Tg (Fig. 4d) mice on day 28 after s.c. infection with RE378. In addition, the destruction of articular cartilage was clearly presented in CD46 Tg mice (Fig. 4c, e) and not in non-Tg mice (Fig. 4d). These observations suggest that the s.c. infection with RE378 could induce osteoclastic bone resorption in the ankle joints of CD46 $\mathrm{Tg}$ mice. 

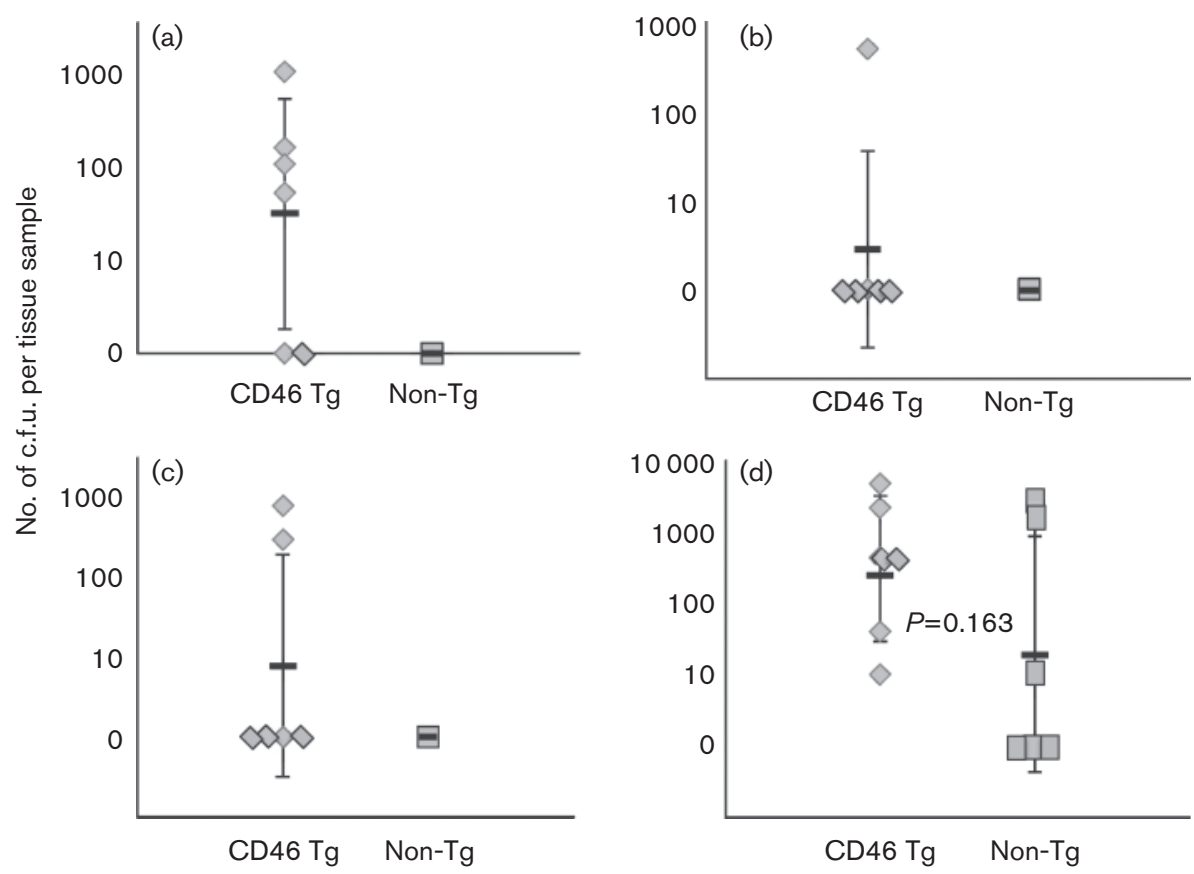

Fig. 2. Bacterial counts in tissues of CD46 Tg and non-Tg mice. CD46 Tg and non-Tg mice were subcutaneously infected with $1 \times 10^{7}$ c.f.u. RE378 per footpad. On day 3 after s.c. infection, the numbers of viable bacteria in each of four tissue samples, liver (a), spleen (b), kidneys (c) and PLNs (d) were determined by plating. The data represent the mean values (horizontal bands) of the number of bacteria per tissue sample \pm SD (vertical bars). Each group had six mice.
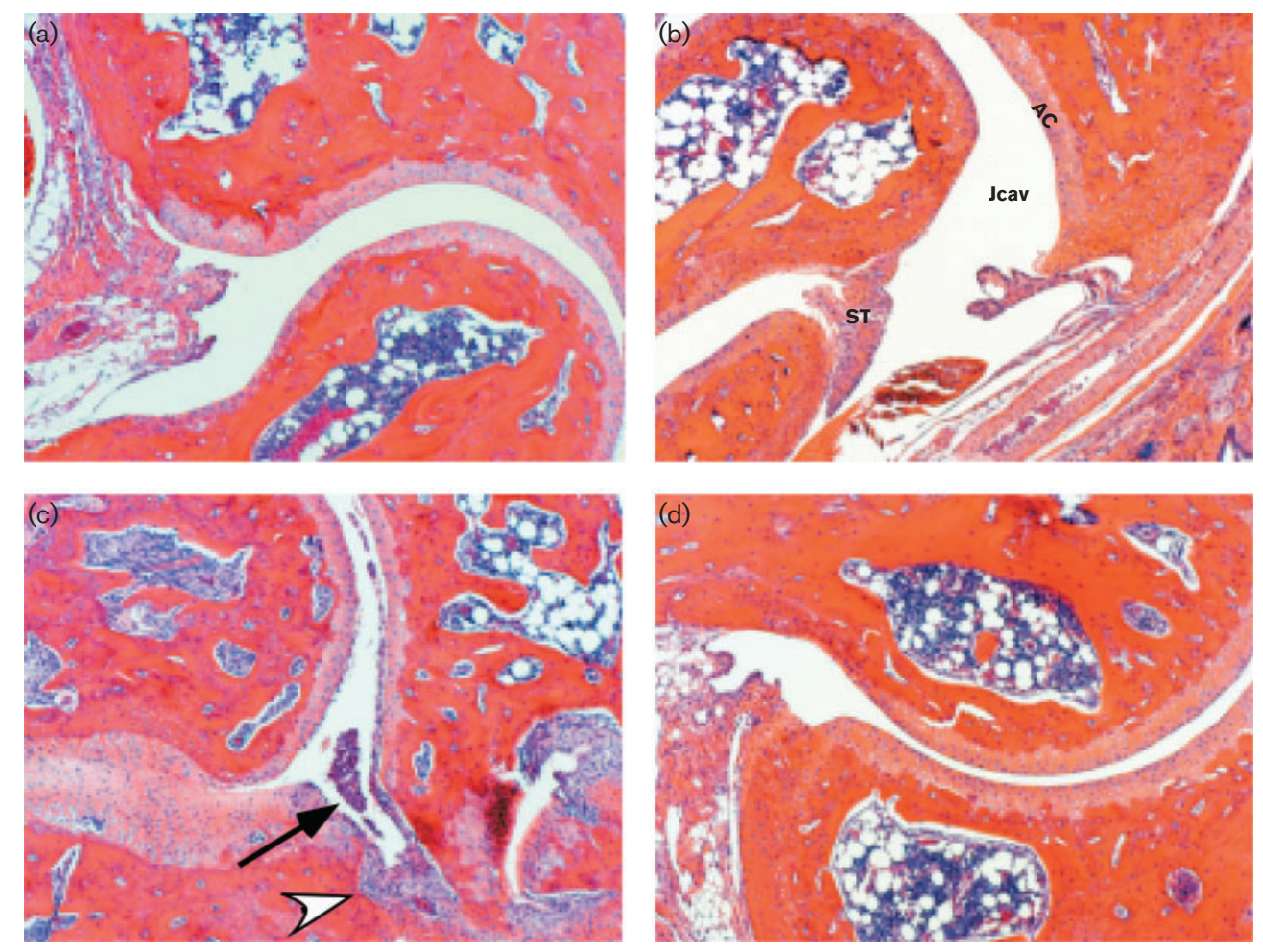

Fig. 3. Histological findings of ankle arthritis of CD46 $\mathrm{Tg}$ and non- $\mathrm{Tg}$ mice. Mouse hind ankles were prepared and the tissue sections were stained with H\&E. On day 28 after s.c. infection with $1 \times 10^{7}$ c.f.u. RE378 per footpad, the sections of CD46 Tg (c) and non- $\mathrm{Tg}$ (d) were examined. Uninfected CD46 Tg (a) and non- Tg (b) mouse foot sections are also shown. Original magnification, $\times 40$. AC, Articular cartilage; Jcav, joint cavity; ST, synovial tissue. The black arrow indicates inflammatory cell infiltration. The white arrowhead indicates synovial pannus formation. 

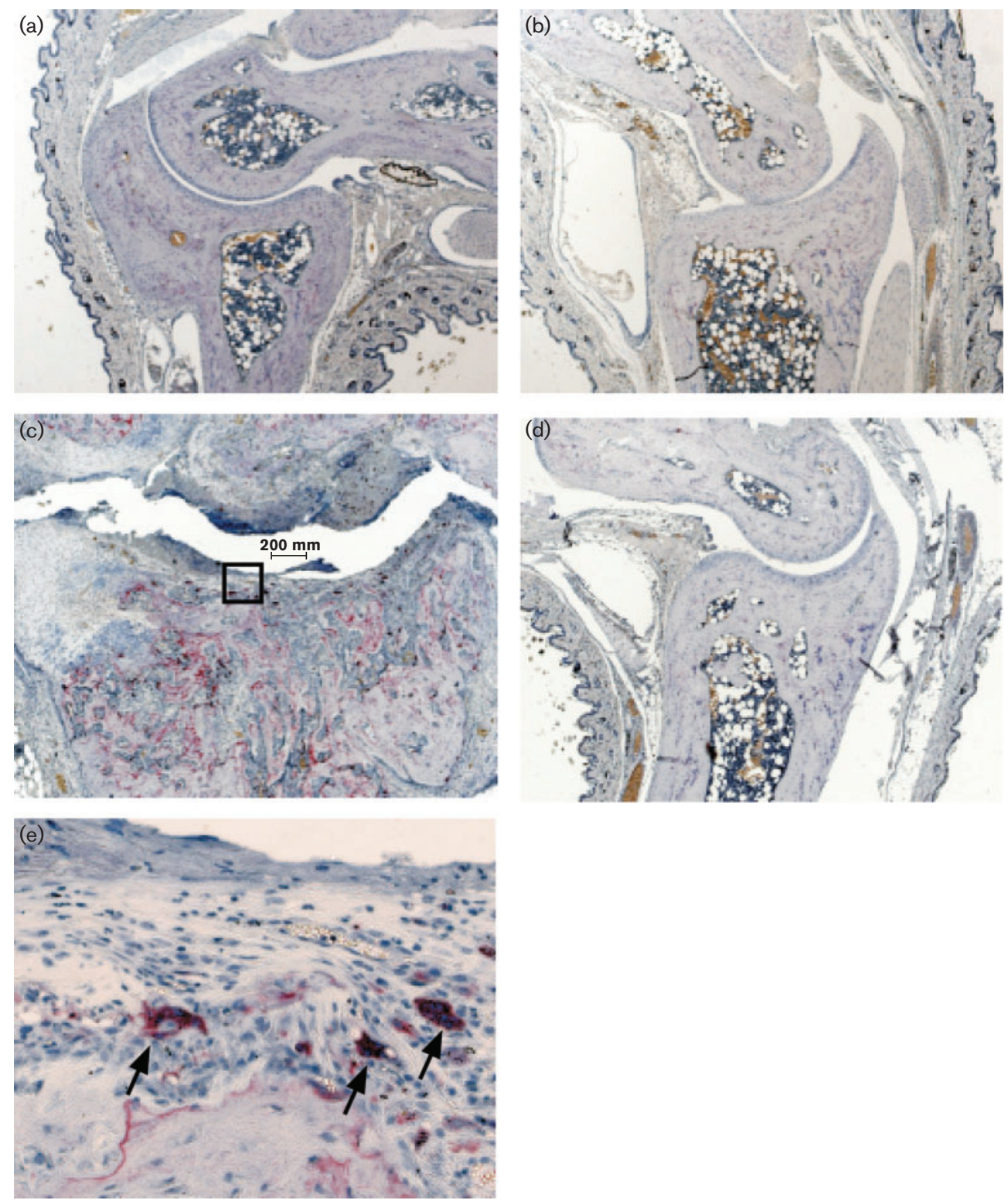

Fig. 4. Expression of multinucleated osteoclasts in the ankles of CD46 Tg and non-Tg mice. Mouse hind ankles were prepared and tissue sections were TRAP stained. On day 28 after s.c. infection with $1 \times 10^{7}$ c.f.u. RE378 per footpad, the sections of CD46 Tg (c, e) and non-Tg (d) were examined. Uninfected CD46 Tg (a) and non- $\mathrm{Tg}$ (b) mouse sections are also shown. (e) A higher-magnification view of the boxed area in (c). Original magnification, $\times 50(a, b, c, d)$ and $\times 400$ (e). The black arrows indicate TRAP-positive osteoclasts.

\section{s.c. infection with RE378 induced the expression of inflammatory and osteoclastogenic cytokines in the foot bones of CD46 Tg mice}

Our results suggest that s.c. infection with RE378 caused prolonged inflammation in the foot joints and bones of CD46 Tg mice, thereby increasingly damaging the area over time. We monitored the mRNA expression levels of OPG, IL-6, RANKL, IL-17A, RANK, TNF- $\alpha$ and IL- $1 \beta$ in the bone samples of the feet of CD46 Tg and non-Tg mice for 25 days after s.c. infection with RE378. As shown in Fig. 5, the mRNA expression levels of the three inflammatory cytokines, namely IL-6 (Fig. 5b), TNF- $\alpha$ (Fig. 5f) and IL- $1 \beta$ (Fig. $5 \mathrm{~g}$ ), were clearly elevated in the foot bones of CD46 $\mathrm{Tg}$ but not in those of non-Tg mice beginning on day 15 $($ TNF- $\alpha$ ) or 25 (IL-1 $\beta$ and IL-6) after s.c. infection with RE378. However, the mRNA expression levels of OPG (Fig. 5a), IL-17A (Fig. 5d), and RANK (Fig. 5e) were not elevated in the foot bones of either CD46 $\mathrm{Tg}$ or non- $\mathrm{Tg}$ mice by s.c. infection with RE378. In contrast, the mRNA expression of RANKL was elevated in the foot bones of 

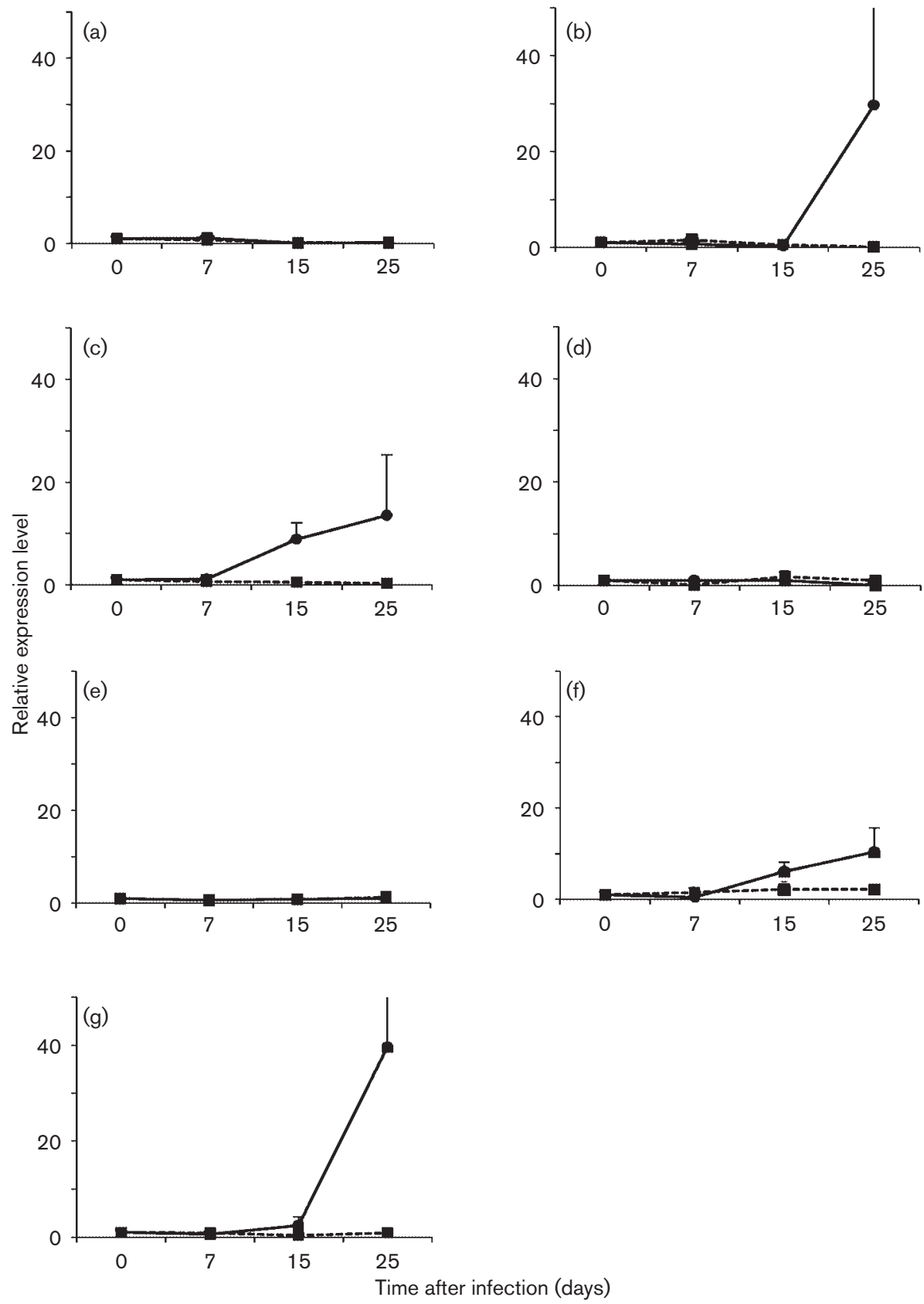

Fig. 5. Comparison of the mRNA expression of OPG, IL-6, RANKL, IL-17A, RANK, TNF- $\alpha$ and IL-1 $\beta$ in the bone samples of the feet of CD46 Tg and non-Tg mice. CD46 Tg ( $\bullet$, solid lines) and non- $\operatorname{Tg}(\boldsymbol{\square}$, dashed lines) mice were subcutaneously infected with $1 \times 10^{7}$ c.f.u. RE378 per footpad. On days $0,7,15$ and 25 after s.c. infection, the total RNA was isolated and purified from the smashed bone sample of the mouse foot. Quantitative real-time RT-PCR was performed as described in Methods. The mRNA expression level of each gene was normalized to the corresponding GAPDH value on each day. Then, each normalized gene value was converted to reflect the amplification of each gene value for day 0 (day $0=1.0$ ). (a) OPG; (b) IL-6; (c) RANKL; (d) IL-17A; (e) RANK; (f) TNF- $\alpha$; (g) IL-1 $\beta$. The data represent the mean values \pm SD $(n=6)$.

CD46 Tg mice but not in those of non-Tg mice from day 15 after s.c. infection with RE378 (Fig. 5c). These data suggest that s.c. infection with S. dysgalactiae subsp. equisimilis could selectively induce the expression of inflammatory cytokines and RANKL.

\section{DISCUSSION}

We previously developed a CD46 Tg mouse model of s.c. infection into both hind footpads with $1 \times 10^{7}$ c.f.u. of the virulent strain of GAS (GAS472) isolated from the blood of 
a patient suffering from streptococcal toxic shock syndrome (Matsui et al., 2009). This mouse model showed progressive $\mathrm{NF}$ in the feet and exponential growth of bacteria in deep tissues, leading to death with a $100 \%$ mortality rate by day 14 after infection (Matsui et al., 2009). Consequently, we had expected that s.c. infection with RE378 would cause a high mortality rate associated with NF in the feet of CD46 $\mathrm{Tg}$ mice. However, against our expectations, the results were far different from those of the CD46 Tg mouse model of GAS infection. The CD46 Tg mice subcutaneously infected with $1 \times 10^{7}$ c.f.u. RE378 into the hind footpads showed a $0 \%$ mortality rate by day 28 after infection and had no severe necrotizing cutaneous lesions in the feet. Indeed, few or no bacteria were colonized in deep tissues of CD46 Tg mice on day 3 after infection, and the bacterial colonization did not persist for 7 days after infection in any tissue sample of CD46 Tg mice (Fig. 2). Based on the complete genome sequencing analysis of $S$. dysgalactiae subsp. equisimilis (Shimomura et al., 2011; S. Y. Murayama \& K. Ubukata, unpublished data), it appears that many virulence factors of $S$. dysgalactiae subsp. equisimilis are closely related to those of GAS, including the twocomponent regulator (CsrR/CsrS), adhesins ( $\mathrm{M}, \mathrm{Fbp}, \mathrm{Cbp}$ and Lbp proteins), factors conferring protection from the host immune system (CepA, GB, GAPDH, Scp and DNase), pore-forming proteins (Hly, SLO, SLS and NADase), transporters (FtsABCD and HtsABC) and others (Hyl and Ska). It seems likely that $S$. dysgalactiae subsp. equisimilis and GAS share the same phage species, and that horizontal gene transfer between $S$. dysgalactiae subsp. equisimilis and GAS has occurred. However, S. dysgalactiae subsp. equisimilis, unlike GAS, does not have erythrogenic toxins (SpeB, SpeG, SpeH, SpeI), streptococcal inhibition of complement-mediated lysis (SIC) or hyaluronan synthesis via has $A B C D$. Therefore, the lethality and major symptoms of $S$. dysgalactiae subsp. equisimilis infection may not correspond with those of GAS infection in the CD46 Tg mouse model of s.c. infection. Furthermore, the results of this study raise the possibility that nonpyogenic arthritis in some patients is attributable to past $S$. dysgalactiae subsp. equisimilis infection. In other words, it is much harder to identify potential arthritis caused by $S$. dysgalactiae subsp. equisimilis infection.

The RANKL/RANK/OPG system is known to be essential for bone and joint remodelling (Boyce \& Xing, 2007a, b; Hamdy, 2007; Ritchlin et al., 2003, 2004). RANK is the cellular receptor of RANKL. OPG provides an alternative binding site for RANKL, and acts as a decoy receptor by blocking RANKL binding to RANK (Yasuda et al., 1998a). RANKL is the primary mediator of osteoclast differentiation, activation and survival (Yasuda et al., 1998b). IL$17 \mathrm{~A}$, in contrast, acts upstream from IL-6 and synergizes with TNF- $\alpha$ to promote inflammation and bone turnover in rheumatoid arthritis (Hot \& Miossec, 2011; Lubberts, 2008, 2010). IL-17A suppresses the expression of bone resorption-related proteinases and osteoclast differentiation (osteoclast precursors into osteoclasts) (Kitami et al.,
2010). The use of the RANKL knockout mouse model has clarified the necessity of RANKL for osteoclast-mediated bone erosion during arthritis (Pettit et al., 2001). Therefore, in the present study RANKL directly contributed to the development of bone damage in response to the expression of inflammatory cytokines. Subsequently, the arthritis would have gradually progressed in the feet of CD46 Tg mice even though the bacteria were cleared from the diseased part.

It has been reported that RANKL is selectively expressed by activated $T$ lymphocytes (Romas et al., 2002; Udagawa, 2003). T lymphocytes can also secrete proresorptive cytokines, including IL-1, IL-6 and IL-17, each of which can stimulate RANKL expression, permitting osteoclast formation by a contact-dependent process (Gillespie, 2007). Meanwhile, CD46 is a ubiquitously expressed type I transmembrane glycoprotein originally identified as a complement-regulatory protein (Kemper et al., 2005; Richards et al., 2007; Wang et al., 2000). $\mathrm{CD} 46$ binds the opsonins $\mathrm{C} 3 \mathrm{~b}$ and $\mathrm{C} 4 \mathrm{~b}$, and functions as a cofactor in their proteolytic degradation (Seya et al., 1986). Human CD46 interacts with at least seven humanspecific pathogens, including GAS. Signalling through CD46 on human T lymphocytes drives them to become regulatory cells, indicating a novel link between the complement system and cellular immunity (Riley-Vargas et al., 2004). In addition, CD46 regulates the cytokine production from T lymphocytes (Cardone et al., 2010). s.c. infection with $S$. dysgalactiae subsp. equisimilis could selectively induce the expression of inflammatory cytokines and RANKL through the function of human CD46 on T lymphocytes. Hence, the CD46 Tg mouse model of infection with $S$. dysgalactiae subsp. equisimilis should be a useful tool in the study of host-pathogen interactions.

\section{ACKNOWLEDGEMENTS}

We are grateful to Mr Osamu Hiraku and Mr Ken Sasaki (Kitasato Institute for Life Sciences, Kitasato University) for their exceptional help with the animal care. This work was financially supported by grants from the Ministry of Health, Labor, and Welfare of Japan under the category 'Research Project for Emerging and Re-emerging Infectious Diseases' (H-19-002, H-20-002, H-21-002, and H-22-013) to K. U. and by a Grant-in-Aid for Young Scientists (B) to H. Y. from the Japan Society for the Promotion of Science.

\section{REFERENCES}

Abdollahi-Roodsaz, S., Joosten, L. A., Helsen, M. M., Walgreen, B., Van Lent, P. L., Van den Bersselaar, L. A., Koenders, M. I. \& Van den Berg, W. B. (2008). Shift from Toll-like receptor 2 (TLR-2) toward TLR-4 dependency in the erosive stage of chronic streptococcal cell wall arthritis coincident with TLR-4-mediated interleukin-17 production. Arthritis Rheum 58, 3753-3764.

Boyce, B. F. \& Xing, L. (2007a). The RANKL/RANK/OPG pathway. Curr Osteoporos Rep 5, 98-104.

Boyce, B. F. \& Xing, L. (2007b). Biology of RANK, RANKL, and osteoprotegerin. Arthritis Res Ther 9 (Suppl. 1), S1. 
Bramhachari, P. V., Kaul, S. Y., McMillan, D. J., Shaila, M. S., Karmarkar, M. G. \& Sriprakash, K. S. (2010). Disease burden due to Streptococcus dysgalactiae subsp. equisimilis (group G and C streptococcus) is higher than that due to Streptococcus pyogenes among Mumbai school children. J Med Microbiol 59, 220-223.

Brandt, C. M. \& Spellerberg, B. (2009). Human infections due to Streptococcus dysgalactiae subspecies equisimilis. Clin Infect Dis 49, 766-772.

Broyles, L. N., Van Beneden, C., Beall, B., Facklam, R., Shewmaker, P. L., Malpiedi, P., Daily, P., Reingold, A. \& Farley, M. M. (2009). Population-based study of invasive disease due to $\beta$-hemolytic streptococci of groups other than A and B. Clin Infect Dis 48, 706712 .

Cardone, J., Le Friec, G., Vantourout, P., Roberts, A., Fuchs, A., Jackson, I., Suddason, T., Lord, G., Atkinson, J. P. \& other authors (2010). Complement regulator CD46 temporally regulates cytokine production by conventional and unconventional T cells. Nat Immunol 11, 862-871.

Davies, M. R., McMillan, D. J., Beiko, R. G., Barroso, V., Geffers, R., Sriprakash, K. S. \& Chhatwal, G. S. (2007). Virulence profiling of Streptococcus dysgalactiae subspecies equisimilis isolated from infected humans reveals 2 distinct genetic lineages that do not segregate with their phenotypes or propensity to cause diseases. Clin Infect Dis 44, 1442-1454.

Gillespie, M. T. (2007). Impact of cytokines and T lymphocytes upon osteoclast differentiation and function. Arthritis Res Ther 9, 103.

Greenspan, A. \& Tehranzadeh, J. (2001). Imaging of infectious arthritis. Radiol Clin North Am 39, 267-276.

Hamdy, N. A. (2007). Targeting the RANK/RANKL/OPG signaling pathway: a novel approach in the management of osteoporosis. Curr Opin Investig Drugs 8, 299-303.

Hot, A. \& Miossec, P. (2011). Effects of interleukin (IL)-17A and IL$17 \mathrm{~F}$ in human rheumatoid arthritis synoviocytes. Ann Rheum Dis 70, $727-732$.

Jonsson, I. M., Pietrocola, G., Speziale, P., Verdrengh, M. \& Tarkowski, A. (2005). Role of fibrinogen-binding adhesin expression in septic arthritis and septicemia caused by Streptococcus agalactiae. J Infect Dis 192, 1456-1464

Joosten, L. A., Abdollahi-Roodsaz, S., Heuvelmans-Jacobs, M., Helsen, M. M., Van den Bersselaar, L. A., Oppers-Walgreen, B., Koenders, M. I. \& Van den Berg, W. B. (2008). T cell dependence of chronic destructive murine arthritis induced by repeated local activation of Toll-like receptor-driven pathways: crucial role of both interleukin-1beta and interleukin-17. Arthritis Rheum 58, 98-108.

Kemper, C., Verbsky, J. W., Price, J. D. \& Atkinson, J. P. (2005). T-cell stimulation and regulation: with complements from CD46. Immunol Res 32, 31-43.

Kitami, S., Tanaka, H., Kawato, T., Tanabe, N., Katono-Tani, T., Zhang, F., Suzuki, N., Yonehara, Y. \& Maeno, M. (2010). IL-17A suppresses the expression of bone resorption-related proteinases and osteoclast differentiation via IL-17RA or IL-17RC receptors in RAW264.7 cells. Biochimie 92, 398-404.

Lövkvist, L., Sjölinder, H., Wehelie, R., Aro, H., Norrby-Teglund, A., Plant, L. \& Jonsson, A. B. (2008). CD46 contributes to the severity of group A streptococcal infection. Infect Immun 76, 3951-3958.

Lubberts, E. (2008). IL-17/Th17 targeting: on the road to prevent chronic destructive arthritis? Cytokine 41, 84-91.

Lubberts, E. (2010). Th17 cytokines and arthritis. Semin Immunopathol 32, 43-53.

Matsui, H., Sekiya, Y., Nakamura, M., Murayama, S. Y., Yoshida, H., Takahashi, T., Imanishi, K., Tsuchimoto, K., Uchiyama, T. \& other authors (2009). CD46 transgenic mouse model of necrotizing fasciitis caused by Streptococcus pyogenes infection. Infect Immun 77, 48064814.

Matsui, H., Sekiya, Y., Takahashi, T., Nakamura, M., Imanishi, K., Yoshida, H., Murayama, S. Y., Takahashi, T., Tsuchimoto, K. \& other authors (2011). Dermal mast cells reduce progressive tissue necrosis caused by subcutaneous infection with Streptococcus pyogenes in mice. J Med Microbiol 60, 128-134.

Maurer, M., Lopez Kostka, S., Siebenhaar, F., Moelle, K., Metz, M., Knop, J. \& Von Stebut, E. (2006). Skin mast cells control T celldependent host defense in Leishmania major infections. FASEB J 20, 2460-2467.

Okada, N., Pentland, A. P., Falk, P. \& Caparon, M. G. (1994). M protein and protein $\mathrm{F}$ act as important determinants of cell-specific tropism of Streptococcus pyogenes in skin tissue. J Clin Invest 94, 965977.

Okada, N., Liszewski, M. K., Atkinson, J. P. \& Caparon, M. (1995). Membrane cofactor protein (CD46) is a keratinocyte receptor for the M protein of the group A streptococcus. Proc Natl Acad Sci U S A 92, 2489-2493.

Perez-Casal, J., Okada, N., Caparon, M. G. \& Scott, J. R. (1995). Role of the conserved C-repeat region of the M protein of Streptococcus pyogenes. Mol Microbiol 15, 907-916.

Pettit, A. R., Ji, H., Von Stechow, D., Müller, R., Goldring, S. R., Choi, Y., Benoist, C. \& Gravallese, E. M. (2001). TRANCE/RANKL knockout mice are protected from bone erosion in a serum transfer model of arthritis. Am J Pathol 159, 1689-1699.

Rantala, S., Vahakuopus, S., Vuopio-Varkila, J., Vuento, R. \& Syrjanen, J. (2010). Streptococcus dysgalactiae subsp. equisimilis bacteremia, Finland, 1995-2004. Emerg Infect Dis 16, 843-846.

Richards, A., Kathryn Liszewski, M., Kavanagh, D., Fang, C. J., Moulton, E., Fremeaux-Bacchi, V., Remuzzi, G., Noris, M., Goodship, T. H. \& Atkinson, J. P. (2007). Implications of the initial mutations in membrane cofactor protein (MCP; CD46) leading to atypical hemolytic uremic syndrome. Mol Immunol 44, 111-122.

Riley-Vargas, R. C., Gill, D. B., Kemper, C., Liszewski, M. K. \& Atkinson, J. P. (2004). CD46: expanding beyond complement regulation. Trends Immunol 25, 496-503.

Ritchlin, C. T., Haas-Smith, S. A., Li, P., Hicks, D. G. \& Schwarz, E. M. (2003). Mechanisms of TNF- $\alpha$ - and RANKL-mediated osteoclastogenesis and bone resorption in psoriatic arthritis. J Clin Invest 111, 821-831.

Ritchlin, C. T., Schwarz, E. M., O’Keefe, R. J. \& Looney, R. J. (2004). RANK, RANKL and OPG in inflammatory arthritis and periprosthetic osteolysis. J Musculoskelet Neuronal Interact 4, 276-284.

Romas, E., Gillespie, M. T. \& Martin, T. J. (2002). Involvement of receptor activator of $\mathrm{NF} \kappa \mathrm{B}$ ligand and tumor necrosis factor $\alpha$ in bone destruction in rheumatoid arthritis. Bone 30, 340-346.

Sakurai, A., Okahashi, N., Nakagawa, I., Kawabata, S., Amano, A., Ooshima, T. \& Hamada, S. (2003). Streptococcus pyogenes infection induces septic arthritis with increased production of the receptor activator of the NF- $\kappa$ B ligand. Infect Immun 71, 6019-6026.

Seya, T., Turner, J. R. \& Atkinson, J. P. (1986). Purification and characterization of a membrane protein (gp45-70) that is a cofactor for cleavage of C3b and C4b. J Exp Med 163, 837-855.

Shimomura, Y., Okumura, K., Murayama, S. Y., Yagi, J., Ubukata, K., Kirikae, T. \& Miyoshi-Akiyama, T. (2011). Complete genome sequencing and analysis of a Lancefield group G Streptococcus dysgalactiae subsp. equisimilis strain causing streptococcal toxic shock syndrome (STSS). BMC Genomics 12, 17.

Sing, A., Trebesius, K. \& Heesemann, J. (2001). Diagnosis of Streptococcus dysgalactiae subspecies equisimilis (group $\mathrm{C}$ streptococci) associated with deep soft tissue infections using 
fluorescent in situ hybridization. Eur J Clin Microbiol Infect Dis 20, 146-149.

Sun, H., Ringdahl, U., Homeister, J. W., Fay, W. P., Engleberg, N. C., Yang, A. Y., Rozek, L. S., Wang, X., Sjöbring, U. \& Ginsburg, D. (2004). Plasminogen is a critical host pathogenicity factor for group A streptococcal infection. Science 305, 1283-1286.

Sylvetsky, N., Raveh, D., Schlesinger, Y., Rudensky, B. \& Yinnon, A. M. (2002). Bacteremia due to $\beta$-hemolytic Streptococcus group G: increasing incidence and clinical characteristics of patients. Am J Med $112,622-626$.

Takahashi, T., Asami, R., Tanabe, K., Hirono, Y., Nozawa, Y., Chiba, N. \& Ubukata, K. (2010a). Clinical aspects of invasive infection with Streptococcus dysgalactiae subsp. equisimilis in elderly patients. J Infect Chemother 16, 68-71.

Takahashi, T., Sunaoshi, K., Sunakawa, K., Fujishima, S., Watanabe, H., Ubukata, K. \& the Invasive Streptococcal Disease Working Group (2010b). Clinical aspects of invasive infections with Streptococcus dysgalactiae ssp. equisimilis in Japan: differences with respect to Streptococcus pyogenes and Streptococcus agalactiae infections. Clin Microbiol Infect 16, 1097-1103.
Takahashi, T., Ubukata, K. \& Watanabe, H. (2011). Invasive infection caused by Streptococcus dysgalactiae subsp. equisimilis: characteristics of strains and clinical features. J Infect Chemother 17, 1-10.

Udagawa, N. (2003). The mechanism of osteoclast differentiation from macrophages: possible roles of $\mathrm{T}$ lymphocytes in osteoclastogenesis. J Bone Miner Metab 21, 337-343.

Wang, G., Liszewski, M. K., Chan, A. C. \& Atkinson, J. P. (2000). Membrane cofactor protein (MCP; CD46): isoform-specific tyrosine phosphorylation. J Immunol 164, 1839-1846.

Yasuda, H., Shima, N., Nakagawa, N., Mochizuki, S. I., Yano, K., Fujise, N., Sato, Y., Goto, M., Yamaguchi, K. \& other authors (1998a). Identity of osteoclastogenesis inhibitory factor (OCIF) and osteoprotegerin (OPG): a mechanism by which OPG/ OCIF inhibits osteoclastogenesis in vitro. Endocrinology 139, 13291337.

Yasuda, H., Shima, N., Nakagawa, N., Yamaguchi, K., Kinosaki, M., Mochizuki, S., Tomoyasu, A., Yano, K., Goto, M. \& other authors (1998b). Osteoclast differentiation factor is a ligand for osteoprotegerin/osteoclastogenesis-inhibitory factor and is identical to TRANCE/RANKL. Proc Natl Acad Sci U S A 95, 3597-3602. 